\title{
ON THE STRUCTURE OF THE FIXED-POINT SET OF A NONEXPANSIVE MAPPING IN A BANACH SPACE
}

\author{
RONALD E. BRUCK, JR.
}

\begin{abstract}
If $C$ is a closed convex subset of a reflexive, strictly convex Banach space $E$, and $T: C \rightarrow E$ is a nonexpansive mapping which has a fixed-point in the interior of $C$, then there exists a nonexpansive mapping $T^{*}: E \rightarrow E$ whose fixed-point set in $C$ is the fixed-point set of $T$.
\end{abstract}

In this note we investigate the structure of the fixed-point set $F(T)$ of a nonexpansive mapping $T: C \rightarrow E$, where $C$ is a closed convex subset of a Banach space $E$ and $T$ does not necessarily map $C$ into itself. It is known that $T$ need not have an extension to a nonexpansive mapping $T^{*}: E \rightarrow \overline{\text { co }} T(C)$, (De Figueiredo and Karlovitz [3], Bruck [1]), so the following result is of interest:

THEOREM. If $C$ is a closed convex subset of a reflexive strictly convex Banach space $E, T: C \rightarrow E$ is nonexpansive, and $T$ has a fixed point in the interior of $C$, then there exists a nonexpansive mapping $T^{*}: E \rightarrow E$ whose fixed points in $C$ are exactly the fixed points of $T$.

Before proving the theorem, we establish a variant of Lemma 5 of [2]:

LemMa. If $y$ is a fixed point of $T$ interior to $C$ then there exists a nonexpansive retraction of $E$ onto the cone

$$
K(y ; F(T))=\mathrm{cl} \cup\{t \cdot F(T)+(1-t) y: t>0\} .
$$

Proof. Let $\delta>0$ be so small that $B=\{x \in E:\|x-y\| \leqslant \delta\}$ is contained in $C$. Since $T y=y$ and $T$ is nonexpansive, $T(B) \subset B$. The restriction $\left.T\right|_{B}$ is a nonexpansive mapping of $B$ into itself, so by [2, Theorem 2] there exists a nonexpansive retraction $r_{1}$ of $B$ onto $F\left(\left.T\right|_{B}\right)=F(T) \cap B$. For $t>0$ define

$$
B_{t}=t B+(1-t) y, \quad F_{t}=t \cdot F(T) \cap B+(1-t) y
$$

and

Received by the editors January 2, 1976.

AMS (MOS) subject classifications (1970). Primary 47H10.

Key words and phrases. Nonexpansive mapping, nonexpansive retraction. 


$$
r_{t}(x)=t \cdot r_{1}\left(t^{-1} x+\left(1-t^{-1}\right) y\right)+(1-t) y
$$

for $x \in B_{t}, r_{t}(x)=y$ for $x \in B_{t} . F(T)$ is convex because $E$ is strictly convex, so for $0<s<t, F_{s} \subset F_{t}$ and $B_{s} \subset B_{t}$. It is easy to verify that $r_{t}$ is a retraction of $E$ into $F_{t}$ which is nonexpansive on $B_{t}$ (but not on $E$ ). Evidently $\cup\left\{B_{t}: t>0\right\}=E$ and $\mathrm{cl} \cup\left\{F_{t}: t>0\right\}=K(y ; F(T))$. Denote $K(y ; F(T))$ by $K$. We shall construct a nonexpansive retraction of $E$ onto $K$ as the limitin an appropriate product space-of a subnet of $\{r: t>0\}$ (limits taken as $t \rightarrow \infty)$.

For $x$ in $E$ put $E_{x}=\{u \in E:\|u-y\| \leqslant\|x-y\|\}, P=\prod_{x \in E} E_{x}$, give $E_{x}$ its weak topology, and $P$ the corresponding product topology. By the reflexivity of $E$ and Tychonoff's theorem, $P$ is compact. Evidently $\left\{r_{t}: t>0\right\}$ is a net in $P$, and therefore has a subnet which converges to some $r$ in $P$.

Given any two $x_{1}, x_{2}$ in $E$, it follows from the weak lower semicontinuity of the norm, the nonexpansiveness of $r_{t}$ on $B_{t}$, and the fact that $x_{1}, x_{2} \in B_{t}$ for sufficiently large $t$, that $\left\|r\left(x_{1}\right)-r\left(x_{2}\right)\right\| \leqslant\left\|x_{1}-x_{2}\right\|$. That is, $r$ is nonexpansive. Since $F_{s} \subset F_{t}$ for $0<s<t, r_{t}$ leaves such point of $F_{s}$ fixed; hence so does $r$. The denseness of $\cup F_{s}$ in $K$ and the continuity of $r$ imply that $r$ fixes each point of $K$. Finally, each $r_{t}$ maps $E$ into $F_{t} \subset K$, and since $K$ is weakly closed (being closed and convex) it follows that the range of $r$ is contained in $K$. These three facts- $r$ fixes each point of $K$, the range of $r$, is contained in $K$, and $r$ is nonexpansive-mean $r$ is a nonexpansive retraction of $E$ onto $K$. Q.E.D.

Proof of Theorem. Put $R=\bigcap\{K(y ; F(T)): y \in F(T) \cap$ int $C\}$. By hypothesis, $F(T) \cap$ int $C \neq \varnothing$, and by the Lemma, there exists a nonexpansive retraction of $E$ onto each $K(y ; F(T))$; by [2, Theorem 5], therefore, there exists a nonexpansive retraction of $E$ onto $R$. Let $T^{*}$ be such a retraction. We claim that $T^{*}$ satisfies the conclusion of the Theorem. Obviously $F(T)$ $\subset C \cap R=F\left(\left.T^{*}\right|_{C}\right)$. If $F(T) \neq F\left(\left.T^{*}\right|_{C}\right)$, let $x_{0} \in C \cap R \backslash F(T)$. We reach a contradiction as follows: let $y_{0} \in F(T) \cap$ int $C$. Since $F(T)$ is closed, the intersection of the line segment $\left[x_{0}, y_{0}\right]$ with $F(T)$ contains a point $z_{0}$ closest to $x_{0} . z_{0} \neq x_{0}$ (because $\left.x_{0} \notin F(T)\right), x_{0} \in C$, and $y_{0} \in$ int $C$, therefore $z_{0} \in$ int $C$. Choose a point $x \neq z_{0}$ in $\left[x_{0}, z_{0}\right]$ which is closer to $z_{0}$ than to bdry $C$; thus $x \notin F(T)$. Now let $y$ be the point of $F(T)$ which is closest to $x$ (this exists because $F(T)$ is closed and convex and $E$ is reflexive). Since $x$ is closer to $z_{0}$ (which is in $F(T)$ ) than to bdry $C, y$ must lie in int $C$. But $R$ is convex, $x_{0}, z_{0} \in R$, and $x \in\left[x_{0}, z_{0}\right]$, so $x \in R$. In particular,

$$
x \in K(y ; F(T)) .
$$

To summarize, there exists a point $x$ such that $x \notin F(T)$, but for the point $y$ of $F(T)$ closest to $x, x \in K(y ; F(T))$. This is an obvious impossibility, and establishes $F\left(\left.T^{*}\right|_{C}\right)=F(T)$. Q.E.D.

Corollary. If, in addition to the hypotheses of the Theorem, $F(T) \subset$ int $C$, then $F\left(T^{*}\right)=F(T)$. 
R. E. BRUCK, JR.

Proof. $F\left(T^{*}\right)$ is convex and, by the Theorem, $F\left(T^{*}\right) \cap C=F(T)$ $\subset$ int $C$; thus $F\left(T^{*}\right) \subset C$, hence $F\left(T^{*}\right)=F(T)$. Q.E.D.

\section{REFERENCES}

1. R. E. Bruck, Jr., A characterization of Hilbert space, Proc. Amer. Math. Soc. 43 (1974), 173-175. MR 49 \#5788.

2. - Properties of fixed point sets of nonexpansive mappings in Banach spaces, Trans. Amer. Math. Soc. 179 (1973), 251-262. MR 48 \#2843.

3. D. G. de Figueiredo and L. A. Karlovitz, On the extension of contractions on normed spaces, Proc. Sympos. Pure Math., vol. 18, Part 1, Amer. Math. Soc., Providence, R. I., 1970, pp. 95-104. MR 43 \#877.

Department of Mathematics, University of Southern California, Los Angeles, CaliFORNIA 90007 